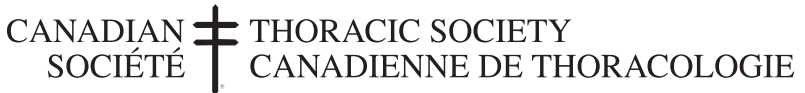

\section{CANADIAN THORACIC SOCIETY} ANNUAL RECEPTION

Plans are underway for this year's Canadian Thoracic Society (CTS) Reception to be held during the annual American Thoracic Society conference in San Diego, California. Please mark your calendars for Saturday, May 21, 6:30 pm to 9:30 pm at Hotel del Coronado.

\section{CTS ANNUAL SCIENTIFIC PROGRAM}

The "Canadian Days" at the American College of Chest Physicians' CHEST conference will follow a new format this year running on a Monday afternoon/evening and a Tuesday morning, rather than a Sunday. Please mark you calendars for Monday, October 31, and Tuesday, November 1, 2005. The Scientific Program is in development and details will be announced once plans are finalized.

\section{CTS MEMBERSHIP}

The CTS membership year is April 1 through March 31. Membership renewal forms will be distributed shortly. Where possible, forms will be distributed via email.

Members of the CTS include specialists in respiratory diseases as well as other physicians, surgeons, medical practitioners and scientists from Canada, the United States and abroad. The Society offers its members the opportunity to learn and collaborate with colleagues in North America and abroad. Members work together to promote the prevention and treatment of respiratory diseases through leadership, education, research and communication.

MOVING? PLEASE LET US KNOW - CONTACT:
Canadian Thoracic Society
3 Raymond Street
Suite 300
Ottawa, Ontario K1R 1A3
Telephone: 613-569-6411 ext 229
Fax: 613-569-8860
E-mail: dsmith@lung.ca

\section{RÉCEPTION ANNUELLE DE LA SOCIÉTÉ CANADIENNE DE THORACOLOGIE}

Les projets sont en cours en vue de la réception de la Société canadienne de thoracologie (SCT) qui aura lieu au congrès annuel de l'American Thoracic Society à San Diego, en Californie. Réservez votre samedi 21 mai, entre 18 h 30 et 21 h 30, à l'hôtel del Coronado.

\section{PROGRAMME SCIENTIFIQUE ANNUEL DE LA SCT}

Les « journées canadiennes » du congrès CHEST de l'American College of Chest Physicians respecteront une nouvelle structure cette année, ayant lieu le lundi en après-midi et en soirée et le mardi matin plutôt que le dimanche. Prévoyez donc y assister le lundi 31 octobre et le mardi $1^{\mathrm{er}}$ novembre 2005 . Le programme scientifique est en cours d'élaboration, et il sera annoncé lorsqu'il sera définitif.

\section{L'ADHÉSION À LA SCT}

L'année d'adhésion à la SCT est du $1^{\mathrm{er}}$ avril au 31 mars. Les formulaires de cotisation seront distribués sous peu, par courriel, dans la mesure du possible.

Les membres de la SCT se composent de spécialistes des maladies respiratoires ainsi que d'autres médecins, chirurgiens, dispensateurs de soins et scientifiques du Canada, des États-Unis et de l'étranger. La Société offre à ses membres l'occasion d'apprendre et de collaborer avec des consœurs et confrères d'Amérique du Nord et de l'étranger. Les membres travaillent ensemble à promouvoir la prévention et le traitement des maladies respiratoires par le leadership, la formation, la recherche et les communications.

VOUS DÉMÉNAGEZ? VEUILLEZ NOUS EN AVISER : Société canadienne de thoracologie 3, rue Raymond Bureau 300 Ottawa ON K1R 1A3

Téléphone : 613 569-6411, post 229

Télécopieur : 613 569-8860

Courriel : dsmith@lung.ca 


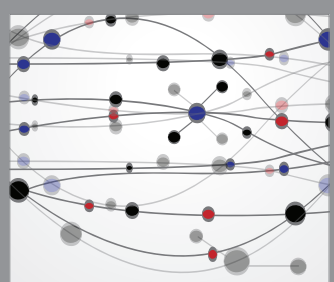

The Scientific World Journal
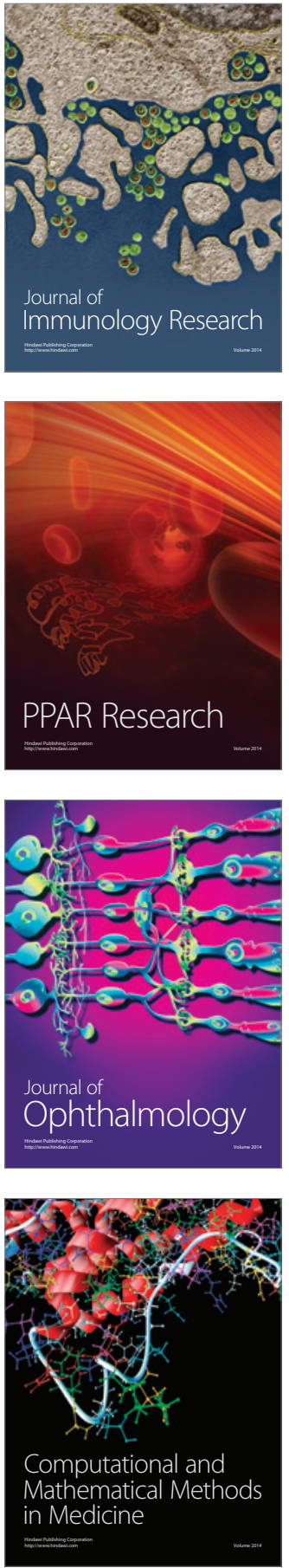

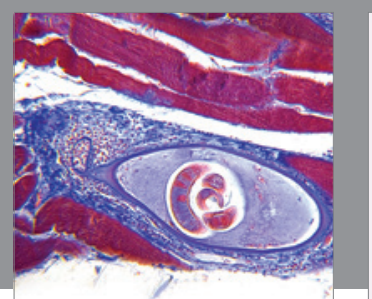

Gastroenterology Research and Practice

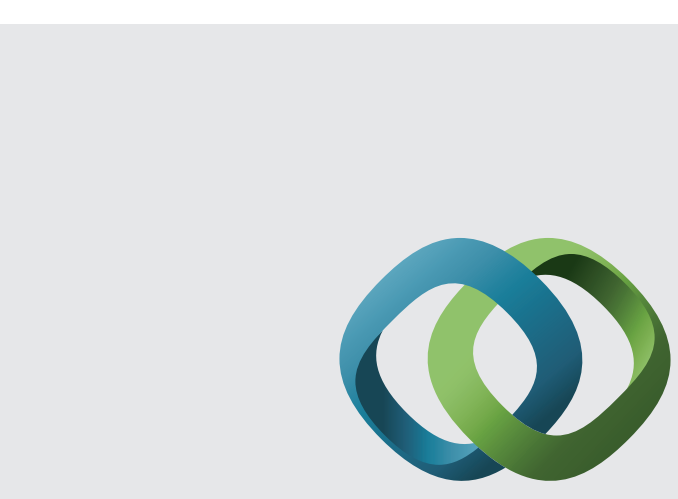

\section{Hindawi}

Submit your manuscripts at

http://www.hindawi.com
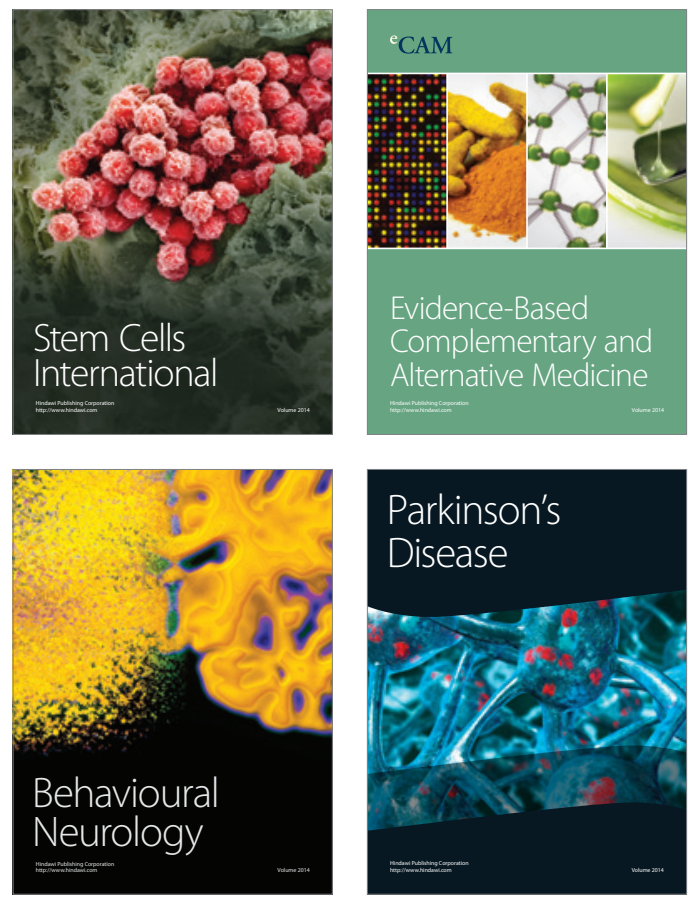
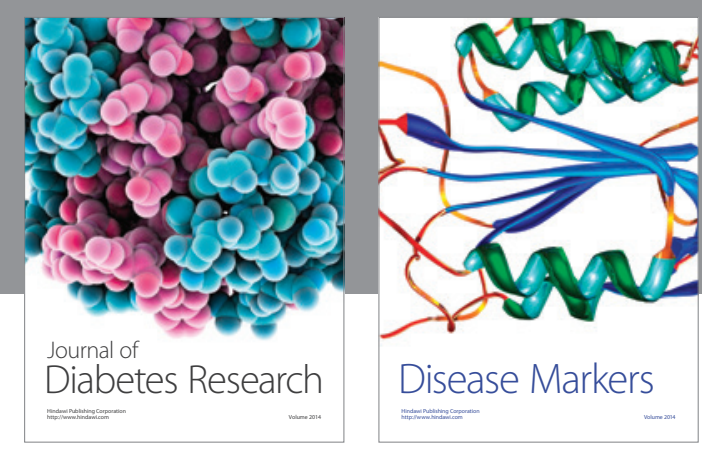

Disease Markers
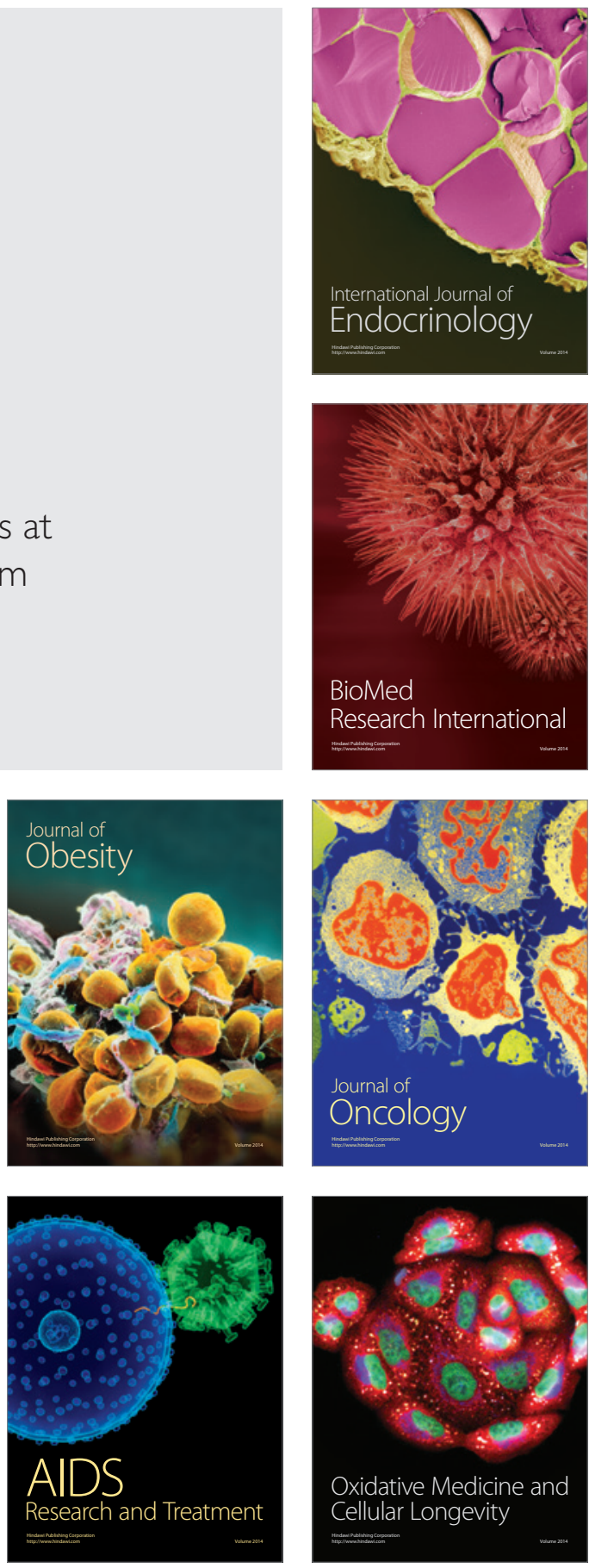